\title{
Research on the rule and algorithm of the transitive judgment of binary relation in a Finite Set
}

\author{
Yingchun $\mathrm{Li}^{1, \mathrm{a}}$,Junfeng $\mathrm{Li}^{2, \mathrm{~b}}$
}

${ }^{1}$ School of Software,University of Science and Technology Liaoning,Anshan, 114051, China

${ }^{2}$ The Cold Rolling Plant of Ansteel co.,Ltd. Liaoning Anshan, 114021, China

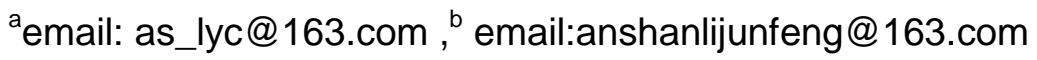

Keywords: Transitivity; Algorithm; Binary relation; Finite Set

Abstract. It sometimes is hard to verify whether a binary relation is transitive by using its definition directly given by , On the basis of analysis and research of the definition of the transitive property of binary relation, this paper launches a new judgment rule, which is very helpful for students to judge the transitivity of a binary relation, at the same time.A judgment algorithm is also presentd.

\section{Introduction}

Binary relation is an important basic concepts in discrete mathematics, is used to discuss the relationship between the two mathematical objects.it is a subset of the cartesian product of set $\mathrm{A}$ and set $B$, and a set of Ordered pair That can be defined as $R=\{\langle x, y\rangle \mid x \in A, y \in B\}$, And it is called a binary relation from set $A$ to set $B[1][2]$, it is also called a binary relation on $A$ when A equals B. The property of binary relations includes reflexive, reflexive, symmetry, antisymmetry and transitivity properties. these properties have a very important role in the practical application of the binary relation,so how to determine whether a binary relation has a certain property has become increasingly important, especially transitive. Compared to other properties, the transitivity is more complex, Its judgment has always been a puzzle for students to learn. Some people have done the relevant research, put forward some ways to judge transitivity[3-6], but these methods are not suitable for classroom teaching. On the basis of analysis and research of the definition of the transitive property of binary relation , this paper launches a new judgment rule, also provides the corresponding implementation algorithm.

\section{Definition of Transitivity for Binary Relation}

The definition[1]: $\mathrm{R}$ is a binary relation defined on the set A, a,b,c are Elements of set $\mathrm{A}$. if there are $<$ a,b $>\in R$ and $<$ b,c $>\in R$, then must be $<a, c>\in R$, we can sure $R$ is transitive.

With the definition to prove the transitive of some binary relation directly is more convenient.

Case 1 try to prove the binary relation " $\leqslant$ ”on real set has transitiveproperty.

Solution: For arbitrary a,b,c $\in \mathrm{R}$, if there are $\mathrm{a} \leqslant \mathrm{b}$ and $\mathrm{b} \leqslant \mathrm{c}$, there must be $\mathrm{a} \leqslant \mathrm{c}$, so we can sure the binary relation " $\leqslant$ ”based on real set with transitivity. In the example above, the given binary relation are used to defined on an infinite setamiliar , and it's a familiar operation.In this situation , using definition to prove transitivity of binary relation is more effective.

However, when a binary relation is given in the form of a finite set of ordered pairs, We can only use the definition to check one by one . Due to the ambiguity of the definition, there are situations in which we cannot make the right judgments. The following example is used to illustrate.

Case $2 A=\{1,2,3,4,5,6\}, R$ is a binary relation defined on the set $A, R=\{\langle 1,1>,\langle 1,2\rangle$,

$<1,3>,<2,3>\}$, Please determine whether the $\mathrm{R}$ is transitive.

Solution: for ordered pairs $\langle 1,1>,<1,2>$, there is $\langle 1,2>\in \mathrm{R}$; for ordered pairs $\langle 1,1>,<1,3>$, there is $<1,3>\in \mathrm{R}$; for ordered pairs $<1,2>,<2,3>$, there is $<1,3>\in \mathrm{R}$; But for ordered pairs 
$<1,3>$ and $<2,3>$, because there is no $<3, \mathrm{x}>\in \mathrm{R}(\mathrm{x} \in \mathrm{A})$, We can't judge according to the definition. So we need to study the definition and give the decision rules which are suitable for this situation. In the analysis, we will use connectives of conditi in propositional logic.

\section{Using Connectives of Condition to Analyze the Transitive Definition}

Connectives are important part of mathematical logic, which is used to link the proposition into a more complex compound proposition. Negation connectives, conjunctive connectives, disjunctive connectives, conditional connectives and Double Conditional connectives are commonly used in mathematical logic . conditional conjunctions is Corresponding to natural language in "if...... Then....." , its symbol is " $\rightarrow$ ". "P $\rightarrow Q$ " read as "if $P$ then $Q$ ".The truth table as shown in table1.From this table we can know that a conditional sentence is false only if its antecedent is false and its consequent is true[1].

Table 1 The truth table for connectives of condition

\begin{tabular}{|c|c|c|}
\hline $\mathrm{P}$ & $\mathrm{Q}$ & $\mathrm{P} \rightarrow \mathrm{Q}$ \\
\hline $\mathrm{T}$ & $\mathrm{T}$ & $\mathrm{T}$ \\
\hline $\mathrm{T}$ & $\mathrm{F}$ & $\mathrm{F}$ \\
\hline $\mathrm{F}$ & $\mathrm{T}$ & $\mathrm{T}$ \\
\hline $\mathrm{F}$ & $\mathrm{F}$ & $\mathrm{T}$ \\
\hline
\end{tabular}

As we mentioned above, the definition of the transitives property for the binary relation is a compound proposition, and the transitives that are used is" $\rightarrow$ ”.

Making the proposition into symbol: Set $\mathrm{R}(\mathrm{x}, \mathrm{y}):<\mathrm{x}, \mathrm{y}>\quad \mathrm{R}$ original definition can sign into that $\forall x \forall y \forall z(R(x, y) \wedge \boldsymbol{R}(y, z) \rightarrow \boldsymbol{R}(x, z)) \quad(\mathrm{x} \in \mathrm{A}, \mathrm{y} \in \mathrm{A}, \mathrm{z} \in \mathrm{A}), \quad \mathrm{R} \quad(\mathrm{x}, \mathrm{y})$

the antecedent of the conditional sentence, $\mathrm{R}(\mathrm{x}, \mathrm{z})$ is the consequent,Table 2 is a truth table for this proposition.

Table 2 the truth table for transitive definition

\begin{tabular}{|c|c|c|}
\hline $\mathrm{R}(\mathrm{x}, \mathrm{y}) \wedge \mathrm{R}$ & $\mathrm{R}(\mathrm{x}, \mathrm{z})$ & $\mathrm{R}(\mathrm{x}, \mathrm{y}) \wedge \mathrm{R}(\mathrm{y}, \mathrm{z}) \rightarrow \mathrm{R}(\mathrm{x}, \mathrm{z})$ \\
\hline $\mathrm{T}$ & $\mathrm{T}$ & $\mathrm{T}$ \\
\hline $\mathrm{T}$ & $\mathrm{F}$ & $\mathrm{F}$ \\
\hline $\mathrm{F}$ & $\mathrm{T}$ & $\mathrm{T}$ \\
\hline $\mathrm{F}$ & $\mathrm{F}$ & $\mathrm{T}$ \\
\hline
\end{tabular}

We can see from the table that conditional sentence is true when its antecedent is false , No matter what the value of the consequent o In mathematical logic, this is called good faith inference. That means in a binary relation $\mathrm{R}$, if only $<x, y>\in R$, but does not exist $<y, z>\in R$, this situation does not affect the transitive of $\mathrm{R}$. A conditional sentence is false only if its antecedent is false and its consequent is true. A binary relation $\mathrm{R}$ is not transitive if and only if that there are $<x, y>\in R,<y$, $\mathrm{z}>\in \mathrm{R}$, but there is no $<\mathrm{x}, \mathrm{z}>\in \mathrm{R}$.

New judgment rule:

$\mathrm{R}$ is a binary relation defined on the set $\mathrm{A}$, We take every ordered pair from $\mathrm{R}$, and use $<\mathrm{a}, \mathrm{b}>$ to represent,do the thing as follow:

(1) if $<b, x>$ exists in $R(x$ is any element in $A)$, and at the same time, $<a, x>$ also exist in $R$,the $<b, x>$ can be sure passed the check,and then the search will be on for new $<b, x>$ until the last one; if $<\mathrm{a}, \mathrm{X}>$ not exist in $\mathrm{R}$, This means that it does not pass the check, the whole judgment is over, $\mathrm{R}$ does not have a transitive.

(2) if $<\mathrm{b}, \mathrm{x}>$ not exists in $\mathrm{R}(\mathrm{x}$ is any element in $\mathrm{A})$, we will assume $<\mathrm{a}, \mathrm{b}>$ has passed the check, and take the next ordered pair and repeat to do (1) and (2). 
(3) If all of the ordered pairs are passed the check ,then $\mathrm{R}$ is transitive.

An example about rule: $A=\{1,2,3,4,5,6\}$, $R$ is a binary relation defined on the set $A$, $\mathrm{R}=\{\langle 1,1\rangle,\langle 1,2\rangle,\langle 1,3\rangle,<2,3\rangle\}$, Please determine whether the $\mathrm{R}$ is transitive.

Judgment process:

check $<1,1>$

$<1,1>,<1,1>\rightarrow<1,1>\in \mathrm{R}$ pass

$<1,1>,<1,2>\rightarrow<1,2>\in \mathrm{R} \quad$ pass

$<1,1>,<1,3>\rightarrow<1,3>\in \mathrm{R} \quad$ pass

Check $<1,2>$

$<1,2>,<2,3>\rightarrow<1,3>\in \mathrm{R}$ pass

Check $<1,3>$ not exists $<3, \mathrm{x}>\in \mathrm{R}$ pass

Check $<2,3>$ not exists $<3, \mathrm{x}>\in \mathrm{R}$ pass

The result is : $\mathrm{R}$ is transitive.

\section{An algorithm to determine the transitive property of binary relation}

Let $\mathrm{R}$ is a binary relation defined on the set, and $|\mathrm{A}|=\mathrm{n}$, A can be represented by an array A [n], $\mathrm{R}$ can be expressed as a two-dimensional array $\mathrm{M}$ [n] [n], $\mathrm{M}$ is defined as follows[2].

$$
\mathrm{M}=(\operatorname{mij}) \mathrm{n} \times \mathrm{n}, \operatorname{mij}=\left\{\begin{array}{cc}
1 & <\mathrm{A}[\mathrm{i}], \mathrm{A}[\mathrm{j}]>\in \mathrm{R} \\
0 & <\mathrm{A}[\mathrm{i}], \mathrm{A}[\mathrm{j}]>\notin \mathrm{R}
\end{array}\right.
$$

Define identification variables $s$ whose value represents the final judgment result, the value of $s$ is true means $\mathrm{R}$ is transitive, otherwise it is not transitive.

the specific steps of the algorithm are as follows:

Step 1: s=true;

Step 2: Traversing each row of array A, if the j column of the i row element is 1,go to step 3;

Step 3: For each 1 of the row $\mathrm{j}$ in the array A,if it is in $\mathrm{k}$ column,we need to see whether the value of the element of the i row k column in the array $\mathrm{A}$ is equal to 1 , if the value of the element $\mathrm{m}[\mathrm{i}][\mathrm{k}]$ is not 1 ,set $\mathrm{s}=$ false and go to step 5;

Step 4: Return s;

Step 5: The algorithm will be terminated.

\section{Conclusion}

The classic definition of the transitive property of the binary relation is not suitable to judge transitivity of some binary relation, the new judgment rule that has been given in the paper is simple and practical,especially for binary relation in a finite set in the form of ordered pair set. This rule does not change the original definition,and not rely on any chart, is very suitable for the classroom teaching of discrete mathematics curriculum. The algorithm presented in this paper is also very easy to implement.

\section{References}

[1] Xiaoling Zuo, Weijian Li ,Yongcai Liu. The Discrete Mathematics [M]. Shanghai scientific and technological literature press, 1982: 110-113

[2] Suyun Geng, Wanling Qu,Liang Zhang. The Discrete Mathematics [M]. tsinghua university press, 2014: 3-86

[3] Jian Guo, Mingru Zhao. Research on Judging a Transitive Bbinary- Relation[J]. Journal of Daqing Normal University 2008 28(5) 45-47

[4 ]Peng Wu. Matrix Discriminance of Transitive Binary Relation in a Finite Set[J]. Journal of 
Chengdu University(Natural Science) 2009(2): 123-124

[5] Lixin Dong. On Judging the Transitivity and Antitransitivity of Binary Relation[J]. Journal of Qinghai Normal University(Natural Science Edition) 2014(2):4-7

[6] Sichun Yang, Xiaolin WANG . Research on the Definition of a Transmission Relation[J]. Microcomputer Development 2003 13(10) 88-89 